\title{
Modification of Conductivity and of Mechanical Properties of Electroactive Polymer (EAP) Thin Films by Titanium Ion Implantation
}

Muhamed Niklaus1, Samuel Rosset1, Phillipe Dubois1, Massoud Dadras2, Herbert Shea1

The following document is a post-print of the article published in Material Research Society Symposium Proceeding, Fall 2007, Vol. 1052: Microelectromechanical Systems--Materials and Devices. The original article can be accessed at

http://www.mrs.org/s_mrs/sec_subscribe.asp?CID=11328\&DID=205373\&action=detail 


\title{
Modification of conductivity and stiffness of electroactive polymer (EAP) thin films
} by metal ion implantation

\author{
Muhamed Niklaus ${ }^{(1)}$, Samuel Rosset ${ }^{(1)}$, Philippe Dubois ${ }^{(1)}$, Massoud Dadras $^{(2)}$, Herbert R. Shea ${ }^{(1)}$ \\ (1)Microsystems for Space Technologies Laboratory, Ecole Polytechnique Fédérale de Lausanne (EPFL), Switzerland \\ (2)Institute of Microtechnology, University of Neuchâtel, Switzerland
}

Dielectric electroactive polymer (DEAP) actuators have a very large percentage displacement, often exceeding 100\%, and therefore require compliant electrodes. Macro-scale (cm and larger) devices, often referred to as artificial muscles, have electrodes made of carbon or metal powder or of conductive grease. This electrode fabrication method is not applicable to micro-scale devices for which electrodes patterned with micron resolution are required. Simple evaporation or sputtering of metal electrodes is not suitable due to the 4 orders of magnitude larger Young modulus of the metals compared to the elastomers, and due the fracture of metal films at very small strains.

We have shown [1] that it is possible to create compliant electrodes by implantation of metal ions into poly-dimethylsiloxane (PDMS) elastomers. Low energy (2 to $35 \mathrm{keV}$ ) implantation creates a thin conductive layer a few $\mathrm{nm}$ below the surface of the polymer without forming a continuous metallic layer, and thus without the stiffening expected from a metal layer. Electrical conductivity of a few $\mathrm{k} \Omega$ / square can be reached with less than $1 \mathrm{MPa}$ increase in Young's modulus for doses of order $10^{16}$ ions $/ \mathrm{cm}^{2}$. The implanted polymer electrodes can repeatedly tolerate strain greater than 0.3 without change in conductivity.

In this work we present the influence of the implantation technique, metal ion species, ion energy, and dose on the mechanical and electrical properties of PDMS membranes and films. The field of ion implantation in polymers is largely unexplored.

Two techniques to fabricate electrodes on PDMS were used: Filtered Cathodic Vacuum Arc (FCVA) and Low Energy broad beam Implanter (LEI). Ti was implanted at $5 \mathrm{keV}$ with the FCVA, at $10 \mathrm{keV}$ and $35 \mathrm{keV}$ with LEI. Au was implanted at $5 \mathrm{keV}$ with FCVA.

The higher the energy and the atomic number of the ions, the longer their stopping distance in the polymer and the larger their distribution or straggle. This is illustrated in Fig.1, which is a numerical simulations of ion distribution in PDMS [2], ignoring the effect of metal ions in the 
membrane. Lower implant energies are therefore preferred to obtain the required concentration and hence conductivity with the lowest possible dose.

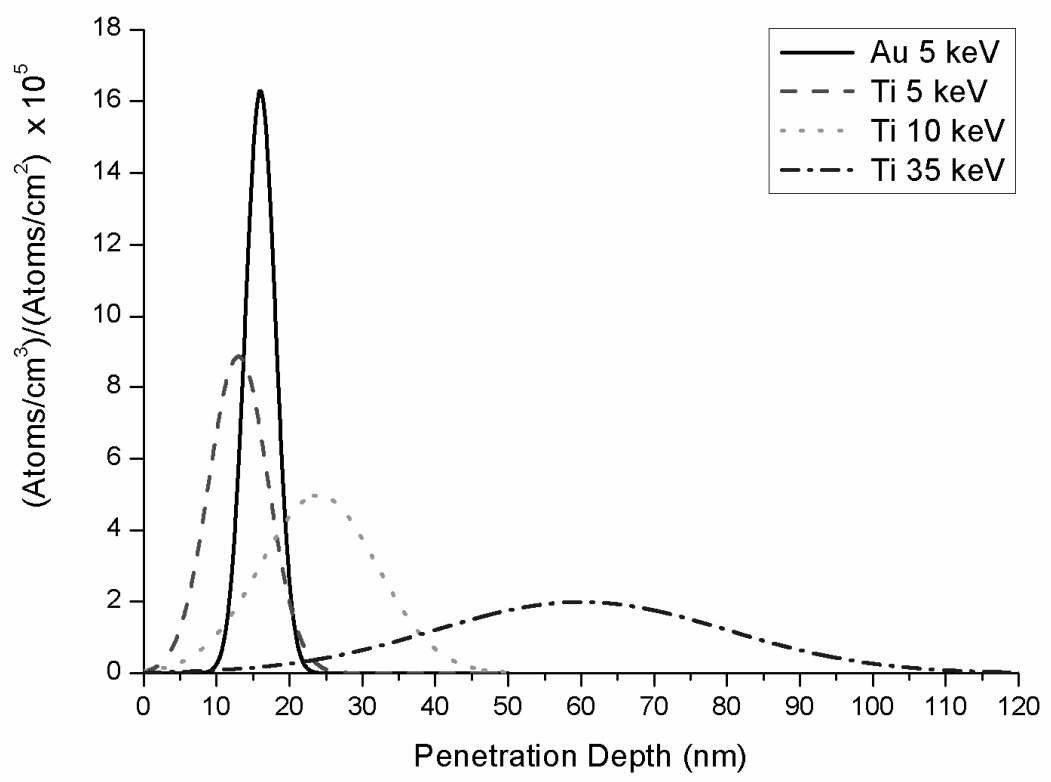

Fig.1. Density vs. penetration depth of Gold and Titanium for different Energies, simulated with TRIM.

Samples for resistivity measurements consist of $25 \mu \mathrm{m}$ thick PDMS films on Si chips. Resistivity is measured on a $5 \times 1 \mathrm{~mm}^{2}$ zone between gold electrodes. For the stress ( $\sigma$ ) and Young modulus (E) measurements a "bulge test" setup was used that measures the deflection of a membrane due to an applied gas pressure. Samples consisted of $25 \mu \mathrm{m}$ thick square and circular PDMS membranes of lateral dimensions 2 to $3 \mathrm{~mm}$.

The doses and energies used were chosen around the region where electrodes are sufficiently conductive (resistivity less than $100 \mathrm{k} \Omega$ /square) yet sufficiently compliant (Young's modulus less than $3 \mathrm{MPa}$ ) that efficient micro-actuators can be fabricated based on them.

The results obtained by FCVA $5 \mathrm{keV}$ Gold implantation are shown in Fig.2. Due to the formation of nano-composites, an increase in membrane stiffness and a decrease of resistivity are observed with increasing dose. Because stable electrodes are required, resistivity as a function of time was measured and shows two regimes (Fig.3): for high conductivity samples the resistivity decreases slightly then stabilizes, and for lower conductivity samples the resistance rapidly increases. Au is well known to form islands when deposited in thin films [3]. The annealing of thin gold films has been extensively studied [4] and occurs for films that exceed a critical thickness. This annealing 
explains the decrease in resistivity observed at higher doses. For thinner discontinuous films, conduction occurs by a number of conduction mechanisms between islands. If the islands coalesce or their spacing increases due to annealing and grain growth, the conductivity of the film will decreases rapidly once the islands are below the percolation threshold. We believe this explains the increases in resistance with time of the low dose samples. For comparison, a dose of $2 \times 10^{16}$ ions $/ \mathrm{cm}^{2}$ corresponds to a film thickness of $3 \mathrm{~nm}$ if all ions were deposited at the surface.

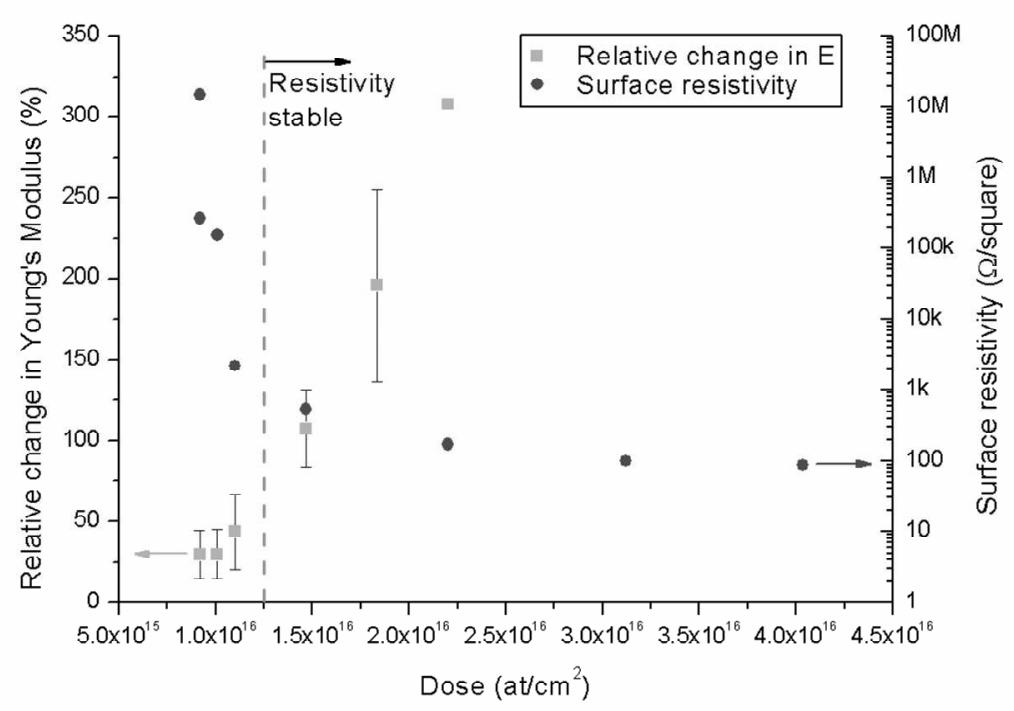

Fig.2.: Surface resistivity and relative Young's modulus vs. ion dose for PDMS films implanted with Au at 5 keV by FCVA. The initial value of the Young's modulus is $0.85 \mathrm{MPa}$.

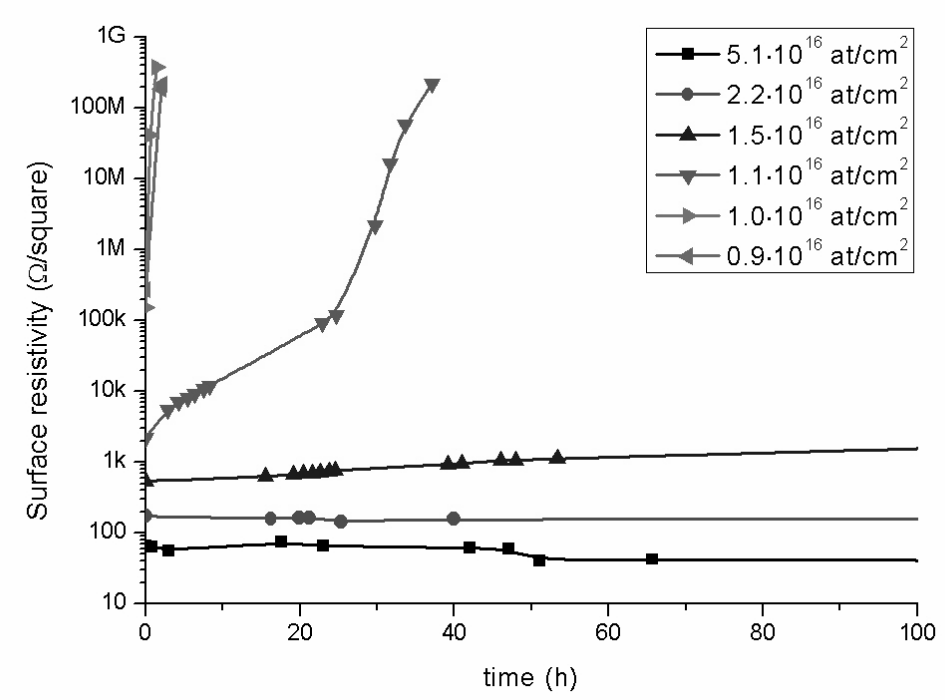

Fig.3: Surface resistivity vs. time for PDMS films implanted with Au $5 \mathrm{keV}$ (in LEI) stored at $100^{\circ} \mathrm{C}$. $\mathrm{High}$ doses lead to metallic films that can be annealed. Low doses lead to island formation and degradation of conductivity with time. 
The resistivity of samples implanted with $\mathrm{Ti}$ at $10 \mathrm{keV}$ by LEI remained unchanged to a few percent after some initial oxidation.

After the successful implantation by FCVA at $5 \mathrm{keV}$, implantation with more traditional implanters was tried. The dose dependence of Young's modulus (E) and resistivity (R) for PDMS membranes implanted with Ti by LEI at $10 \mathrm{keV}$ is shown in Fig.4. The overall trend of $\mathrm{E}$ and $\mathrm{R}$ vs. dose is the same as for $\mathrm{Au}$ at $5 \mathrm{keV}$. To reach similar resistivity an approximately $50 \%$ larger dose is required, consistent with Figure 1. However the Young's modulus is 2 orders of magnitude larger than for $5 \mathrm{keV} \mathrm{Au,} \mathrm{a} \mathrm{much} \mathrm{larger} \mathrm{increase} \mathrm{than} \mathrm{can} \mathrm{be} \mathrm{explained} \mathrm{by} \mathrm{the} \mathrm{slightly} \mathrm{larger} \mathrm{dose}$ if a simple linear superposition model is used.

This difference is not primarily due to the nature of the ion, but to the nature of the implantation process. The FCVA process is pulsed (600 $\mu \mathrm{s}$ pulses every second, $3.7 \times 10^{13}$ ions $\left./ \mathrm{cm}^{2} / \mathrm{pulse}\right)$, probably generates large local heating at every pulse, and requires a total time of order $500 \mathrm{~s}$, whereas the LEI process rasters a continuous $3 \mu \mathrm{A}$ beam over the surface, requiring several hours to complete the implantation. Charging is known to play a large role in the detailed microstructure of the films (see AFM roughness studies below) and the two implantation techniques provide order of magnitude difference in average beam current. Increasing the $\mathrm{Ti}$ ion energy to $35 \mathrm{keV}$ (LEI) gives samples with Young's modulus even higher than at $10 \mathrm{keV}$. FCVA implantaion with Ti at 5 $\mathrm{keV}$ gives results very similar to $5 \mathrm{keV}$ Au by FCVA.

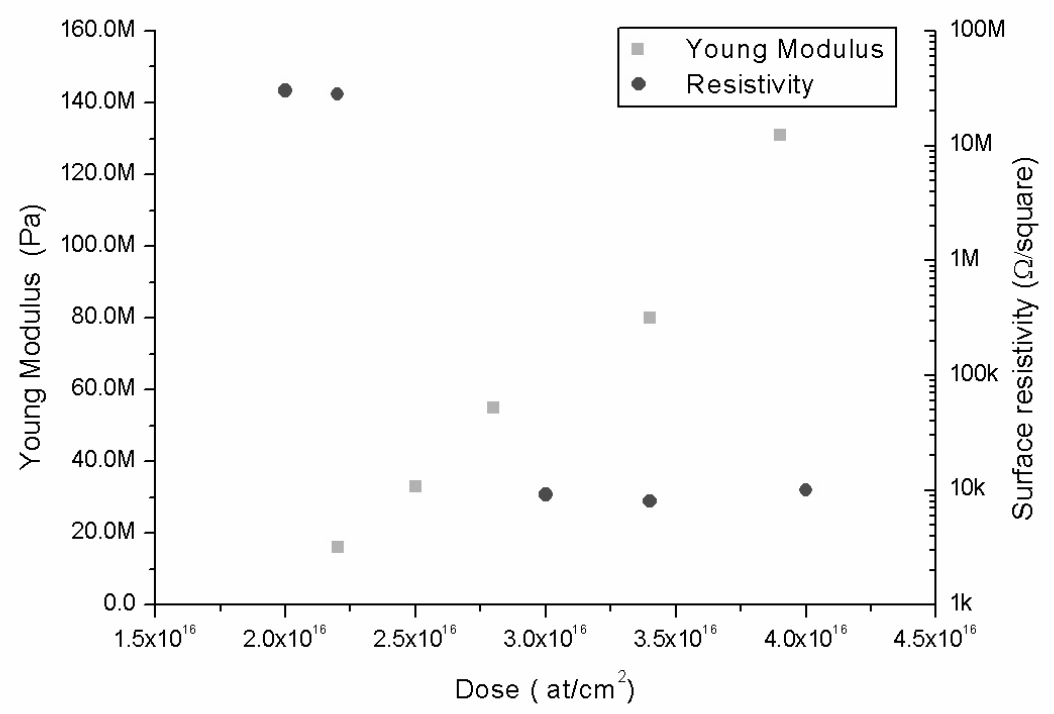

Fig.4. Surface Resistivity and Young's modulus of PDMS films after implantation with Ti at $10 \mathrm{keV}$ by LEI. The initial value of the Young's modulus is $0.85 \mathrm{MPa}$ 
Implantation has a strong influence on surface roughness of polymers due to sputtering, local stress modification, charging and heating. The PDMS films had initial roughness of $3 \mathrm{~nm} \mathrm{rms}$. Figures 5 and 6 show the roughness increase as a function of implant dose and implant technique. The FCVA increase roughness up to $100 \mathrm{~nm}$, while the LEI tool yields films with roughness of over $1 \mu \mathrm{m}$. This is an indication of the dramatic difference in microstructure obtained at comparable doses but with different implanters, which is reflected in the nearly 100 fold difference in Young's modulus of LEI compared to FCVA.

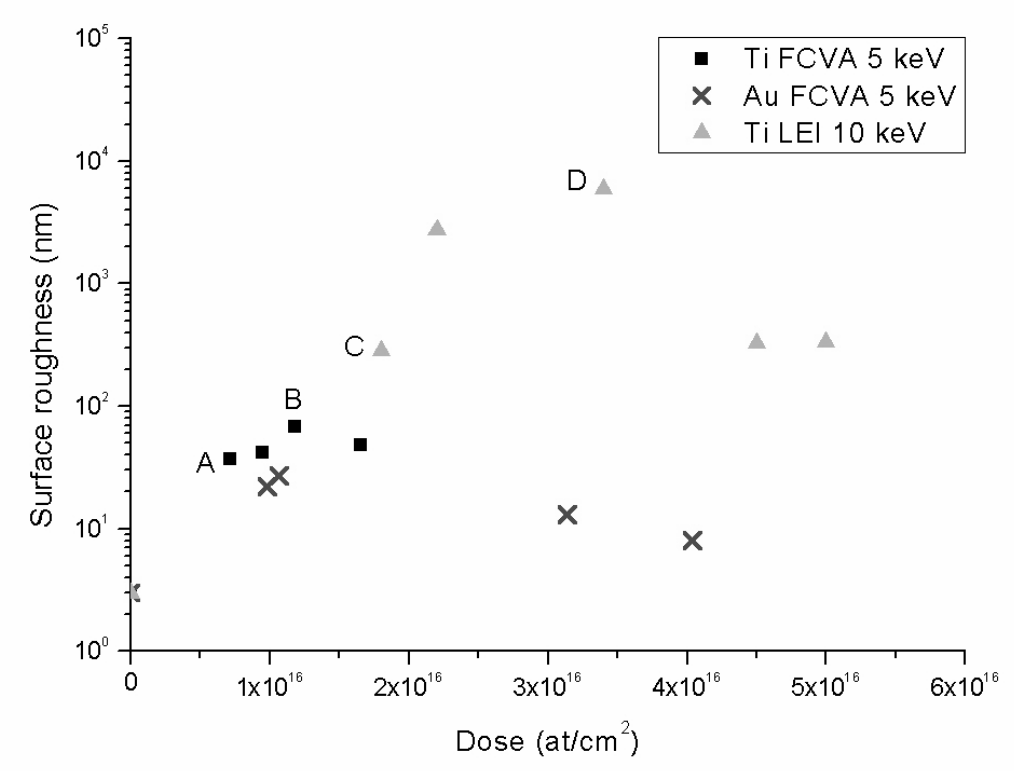

Fig.5. Surface Roughness of PDMS films as a function of ion dose for different implantation methods and ion species. Letters A through D refer to sample shown in more detail in Figure 6. 

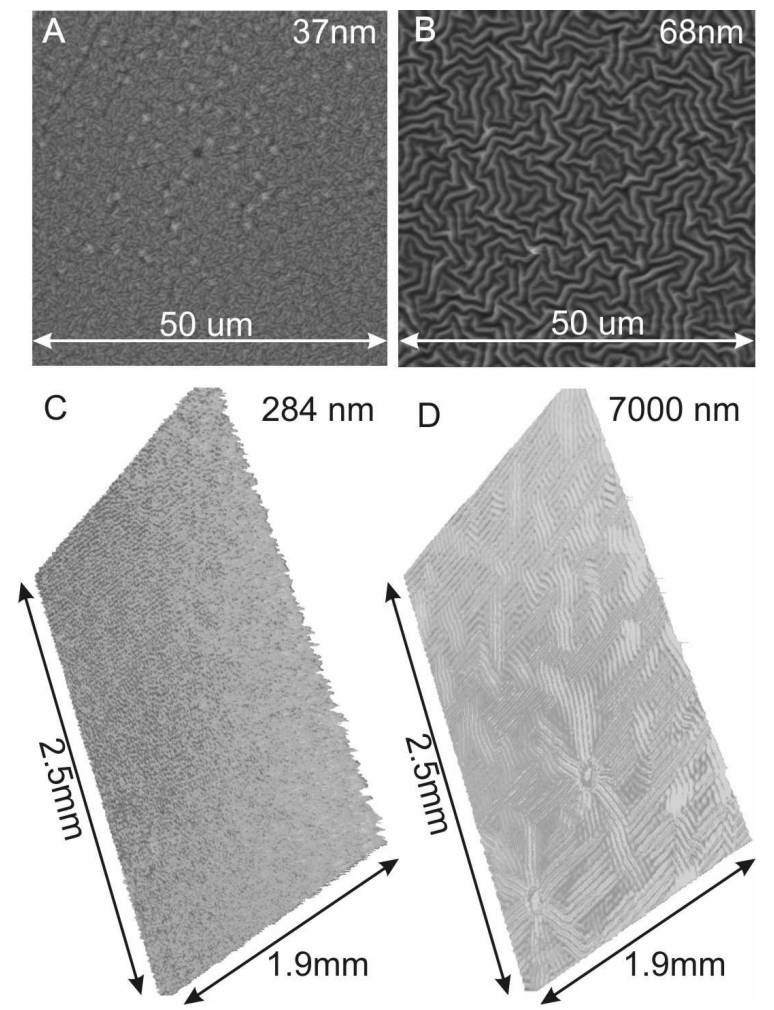

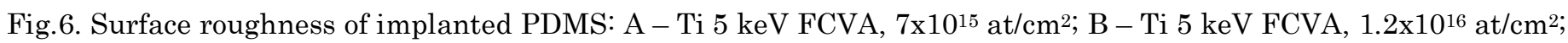

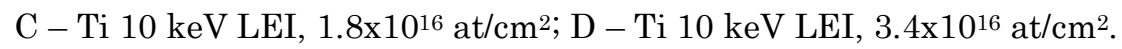

To better understand the modification of physical properties due to the metal ion implantation a special cryogenic preparation technique was developed, tested and used to prepare TEM lamella of implanted PDMS membranes. In TEM transmission mode the arrangement of clusters can be observed, where else in diffraction mode, important information about the crystalline film structure of high dose Ti implanted elastomer is revealed.

This study enabled the definition of the ion dose and energy domain where a stable low resistivity can be obtained simultaneously with limited stiffening of the polymer membrane. To create compliant electrodes, the FCVA type of implanter is much better suited than conventional implanting equipment. We are currently performing more systematic measurements to provide a comparison between different ion species implanted in similar conditions. Our research focuses on the TEM and AFM observation, and modeling of the nano-structure created in the PDMS. 


\section{Acknowledgments:}

This research was supported by the Swiss National Science Foundation grant \# 200021-111841.

The LEI implantations were performed at the Center for Application of Ion Beams in Materials Research at the Forschungszentrum Dresden-Rossendorf, Germany.

\section{References:}

[1] "Microactuators Based On Ion-Implanted Dielectric Electroactive Polymer Membranes (EAP)", P. Dubois et al, Sensors and Actuators A: Physical, Vol. 130-131, pp. 147-154 (2006)

[2] SRIM - The Stopping and Range of Ions in Matter- software at: http://www.srim.org/, J. F. Ziegler \& J. P. Biersack

[3] "Initial growth and morphology of thin Au films on TiO2(110)". Lei Zhang et al., Surface Science 439 (1999) pp.73-85

[4] "Structural and electrical properties of thin d.c. magnetron-sputtered gold films deposited on float glass", Christian Markert et al., Surf. Interface Anal. 2006; 38: pp. 715-718 